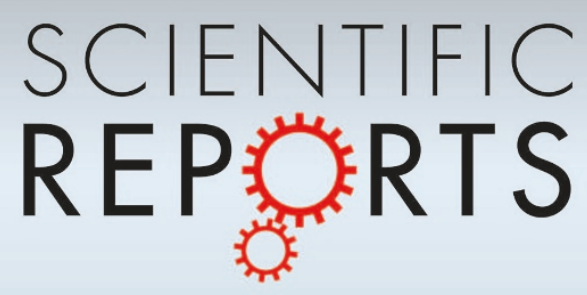

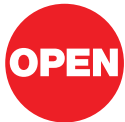

SUBJECT AREAS:

NANOSCALE

BIOPHYSICS

RADIOTHERAPY

NANOPHOTONICS AND

PLASMONICS

NANOTECHNOLOGY IN

CANCER

Received

13 August 2012

Accepted

7 January 2013

Published

27 February 2013

Correspondence and requests for materials should be addressed to

V.P.Z.

(ZharovVladimirP@ vams.edu)

\title{
Photothermal nanodrugs: potential of TNF-gold nanospheres for cancer
} \section{theranostics}

\author{
Jingwei Shao ${ }^{1,2}$, Robert J. Griffin ${ }^{3,4}$, Ekaterina I. Galanzha², Jin-Woo Kim³,6, Nathan Koonce ${ }^{3}$, \\ Jessica Webber ${ }^{3}$, Thikra Mustafa ${ }^{7}$, Alexandru S. Biris ${ }^{7}$, Dmitry A. Nedosekin² \& Vladimir P. Zharov ${ }^{2,4}$ \\ ${ }^{1}$ College of Chemistry and Chemical Engineering, Fuzhou University, Fuzhou, Fujian, 350108, China, ${ }^{2}$ Phillips Classic Laser and \\ Nanomedicine Laboratories, Winthrop P. Rockefeller Cancer Institute, University of Arkansas for Medical Sciences, Little Rock, \\ Arkansas 72205, USA, ${ }^{3}$ Department of Radiation Oncology, University of Arkansas for Medical Sciences, Little Rock, Arkansas \\ 72205, USA, ${ }^{4}$ Arkansas Nanomedicine Center, University of Arkansas for Medical Sciences, Little Rock, AR, 72205, USA, ${ }^{5}$ Bio/ \\ Nano Technology Laboratory, Institute for Nanoscience and Engineering, University of Arkansas, Fayetteville, AR, 72701, USA, \\ ${ }^{6}$ Department of Biological and Agricultural Engineering, University of Arkansas, Fayetteville, AR, 72701, USA, ${ }^{7}$ Center for \\ Integrative Nanotechnology Sciences, University of Arkansas at Little Rock, AR, 72204, USA.
}

Nanotechnology has been extensively explored for drug delivery. Here, we introduce the concept of a nanodrug based on synergy of photothermally-activated physical and biological effects in nanoparticle-drug conjugates. To prove this concept, we utilized tumor necrosis factor-alpha coated gold nanospheres (Au-TNF) heated by laser pulses. To enhance photothermal efficiency in near-infrared window of tissue transparency we explored slightly ellipsoidal nanoparticles, its clustering, and laser-induced nonlinear dynamic phenomena leading to amplification and spectral sharpening of photothermal and photoacoustic resonances red-shifted relatively to linear plasmonic resonances. Using a murine carcinoma model, we demonstrated higher therapy efficacy of Au-TNF conjugates compared to laser and Au-TNF alone or laser with TNF-free gold nanospheres. The photothermal activation of low toxicity Au-TNF conjugates, which are in phase II trials in humans, with a laser approved for medical applications opens new avenues in the development of clinically relevant nanodrugs with synergistic antitumor theranostic action.

$\mathrm{V}$ arious nanoparticles have been extensively explored either as imaging contrast agents or as a transformer of various energy modalities (e.g., laser, ultrasound, and radio-waves) to thermal and accompanied phenomena (e.g., nanobubbles, acoustic and shock waves, or nanoparticle explosion) responsible for therapeutic effects or as vehicles for drug delivery ${ }^{1-11}$. In particular, laser-induced photothermal (PT) effects in targeted cells lead to protein denaturation, mechanical membrane damage, and even cell fragmentation ${ }^{8-10}$. PT effects can also provide controllable drug release from nano-carriers followed by conventional biochemical drug action $^{11}$. However, little progress has been made in the use of nanotechnology to directly activate and/or amplify biochemical drug action.

In this work, we aimed to explore whether a laser-induced PT effects in nanoparticle-drug conjugates can activate biochemical drug action. We tested this concept with in vitro and in vivo models using the cytokine tumor necrosis factor-alpha (TNF) bound to the surface of $\sim 33 \mathrm{~nm}$ gold nanospheres (referred to as Au-TNF). Furthermore, we demonstrated higher therapeutic efficacy of laser at wavelength of $690 \mathrm{~nm}$ with Au-TNF conjugates compared with laser at $532 \mathrm{~nm}$ alone, laser at $690 \mathrm{~nm}$ alone, Au-TNF alone, and laser at $690 \mathrm{~nm}$ with TNF-free nanoparticles. These results suggested new options for synergistic cancer treatment using multiple theranostic modalities with multifunctional nanoparticle-drug conjugates.

\section{Results}

Concept of synergistic physical and biochemical cancer treatment. We developed the concept of the photothermal activated nanodrug based on synergy of physical and biochemical mechanisms. To verify this concept the conjugated with a thermosensitizing cytokine (TNF alpha) were heated by short laser pulses. We hypothesized that the antitumor properties of this Au-TNF conjugate would be enhanced through pulsed PT heating of the gold nanospheres. Depending on laser parameters (e.g., wavelength, energy, and pulse width), PT effects can lead to single phenomenon or spatially and temporary overlapping multiple dynamic phenomena such 
as nanoparticle temperature increase, thermal expansion, explosion and fragmentation as well as a formation of acoustic waves, nanobubbles and microbubbles (Fig. 1). In turn, these phenomena might increase the toxicity of TNF by various mechanisms. The AuTNF conjugates synthesized by loading polyethylene glycol (PEG) coated $33 \pm 3 \mathrm{~nm}$ gold nanospheres with TNF (Fig. 2a) were initially designed to target the delivery of the drug to solid tumors via enhanced permeability and retention ${ }^{12-15}$. Au-TNF has been reported to result in an antitumor response when used in combination with cryosurgery or hyperthermia treatment ${ }^{13-16}$. The $\mathrm{Au}-\mathrm{TNF}$ conjugates were less systemically toxic and more effective in tumor treatment compared to TNF alone. Although nanoparticles have demonstrated improved safety and efficacy, little is known about the synergistic results by taking into account potent thermosensitizing ability of $\mathrm{TNF}^{15}$. To explore the effects of laser on TNF actions, we applied PT therapy with pulsed laser which was previously introduced by our group to kill individual cancer cells targeted by gold nanospheres through laser-induced nanobubbles around the overheated nanoparticles ${ }^{9,10}$. Gold nanoparticles, especially gold nanospheres, are an ideal nanoplatform for development as a PT nanodrug because of simple synthesis procedures, low cost, stable properties, strong optical absorption profiles with low toxicity and ability to be easily conjugated with chemicals ${ }^{17-20}$. In particular, Au-TNF conjugates called AurImmune $^{\mathrm{TM}}$ (referred also as CYT-6091) completed (as a single therapy) a phase I trial in humans and is currently in phase $\mathrm{II}^{21}$.

Nevertheless, plasmon absorption resonances of individual gold nanospheres are located in the spectral range of 520-530 nm where strong blood absorption background makes it difficult to apply PT therapy $^{22-24}$. In particular, the ratio of coefficient absorption $\alpha$ at wavelength of plasmon resonance of bare nanoparticles near 525 and off-resonance at $690 \mathrm{~nm}$ is $\alpha_{525} / \alpha_{690} \mathrm{~nm} \sim 58$ (Supplementary Document 1). We explored several ways to enhance PT effect in near-infrared (NIR) region with minimum tissue and especially blood absorption, where gold nanospheres provides weak plasmonic absorption.

The enhancement of PT effects in far-red and NIR region. Plasmon resonance of ideal 30-nm gold nanospheres appears at $520 \mathrm{~nm}$ and slightly shifts to the longer wavelengths after PEG coating due to an increased index of refraction. Specifically, we determined that absorption maximum for Au-TNF containing
PEG layer red shifted to 535-540 nm (Fig. 2f). By TEM imaging we revealed that $\mathrm{Au}-\mathrm{TNF}$ nanospheres had a relatively large portion (up to $30 \%$ ) of ellipsoidal nanoparticles in the sample (e.g., Fig. 2a) with the ratio of major to minor diameters in the range of 1.1-1.3 (up to 2 for a few percent of the nanoparticles). This resulted in enhancement of off-resonance NIR absorbance with the $\alpha_{525} /$ $\alpha_{690} \mathrm{~nm}$ ratio reduced from 58 to the level of $10-15$.

Secondly, our previous work suggested that $\mathrm{Au}$-TNF may follow the model of nanoparticle self-assembly in living systems ${ }^{10,18}$. Indeed we and others discovered that Au-TNF had a tendency for clustering in both small (Fig. 2b) and large (Fig. 2c-e, Supplementary Fig. S1, S2) clusters in vitro as well as in vivo (Supplementary Fig. S3, S4). This aggregation was accompanied by a red-shift of their plasmonic resonances with simultaneous enhancement of NIR absorption ratio to a level of $\alpha_{525} / \alpha_{690} \mathrm{~nm} \sim 2-3$ (Fig. $2 \mathrm{f}$ ). These plasmonic phenomena allow more effective application of PT therapy in spectral region of minimal blood absorption background.

In addition, we also explored the role of a new phenomenon recently discovered by us relating to nonlinear dynamic processes during short laser pulses ${ }^{25,26}$ leading to dramatic amplification (50100 -fold) and spectral sharpening (up to $\sim 1 \mathrm{~nm}$ ) of PT and photoacoustic (PA) ${ }^{26-29}$ effects. Briefly, at laser energy slightly above the threshold for nanobubble generation, the nanobubbles provide simultaneous amplification and narrowing of the PT/PA spectra near the centers of existing absorption peaks. At even higher energy, we have observed laser spectral burning (PT bleaching) leading to spectral holes and simultaneous off-center new PT/PA ultrasharp spectral resonances ${ }^{25}$. Indeed, for $\mathrm{Au}-\mathrm{TNF}$ conjugates we observed with increased energy fluence a PT/PA signal behavior which is typical for many absorbing objects (e.g., nanoparticles, chromophores and dyes $)^{25}$ : linear and then nonlinear nanobubble-related signal increases, signal saturation, a signal decrease (not shown) and then significant signal increase associated with nanoparticle size and shape modification or enhancement associated with nanoparticle explosion $^{17}$. At $532 \mathrm{~nm}$ nonlinear PT/PA phenomena appeared at lower energy fluence compared to $690 \mathrm{~nm}$ (Figs. $3 \mathrm{a}$ and $3 \mathrm{~b}$ ) due to higher initial stationary Au-TNF absorption. However, with further increase in energy fluence a significant PT/PA effect amplification at $690 \mathrm{~nm}$ made the difference between the PT/PA signals at $532 \mathrm{~nm}$ and $690 \mathrm{~nm}$ lower: just 3-5-fold compared to theoretical difference of 58-fold for ideal nanospheres. As our model predicts ${ }^{25}$ we observed ultrasharp (1-2 nm) PT/PA resonances near absorption maximum

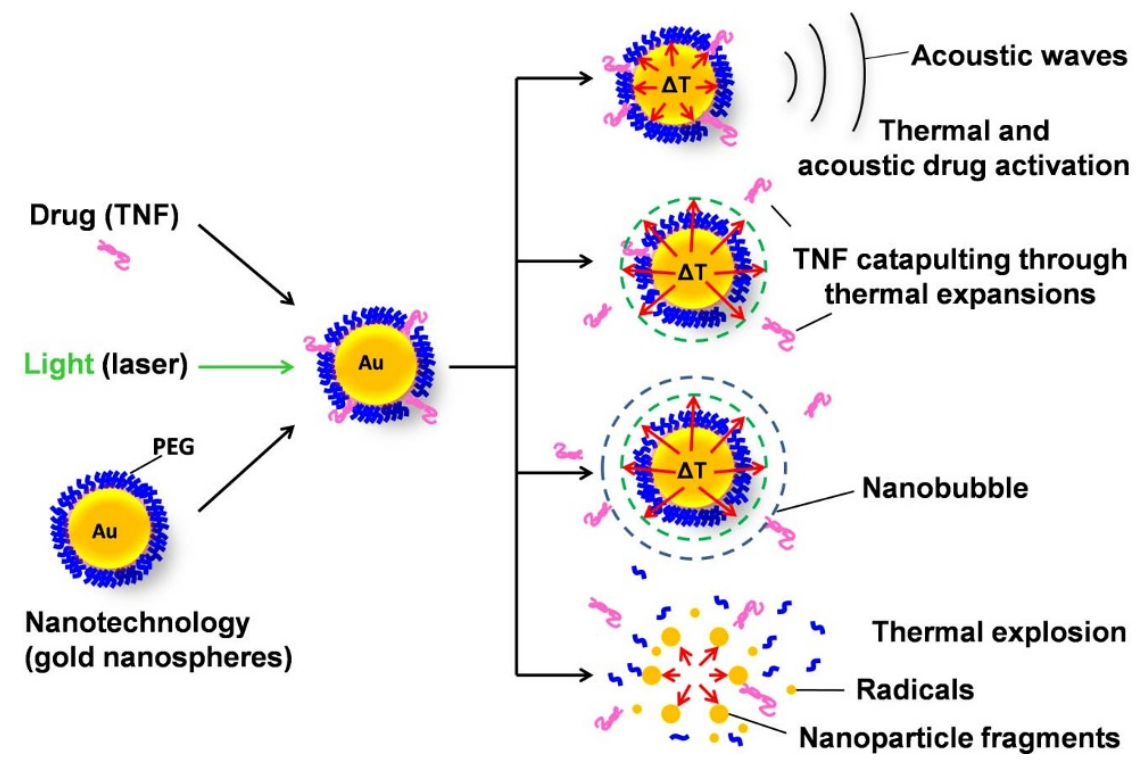

Figure $1 \mid$ Principle of nanodrug action based on PT activation of TNF- gold nanosphere conjugates through controllable temperature increase, thermal expansion, acoustic nanobubble, and explosion phenomena. 
a

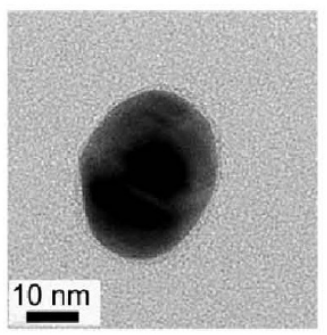

e

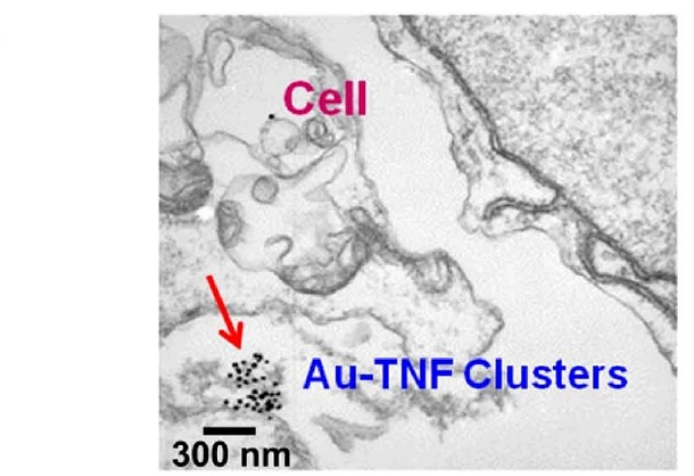

g

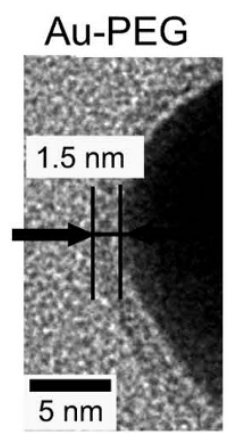

b
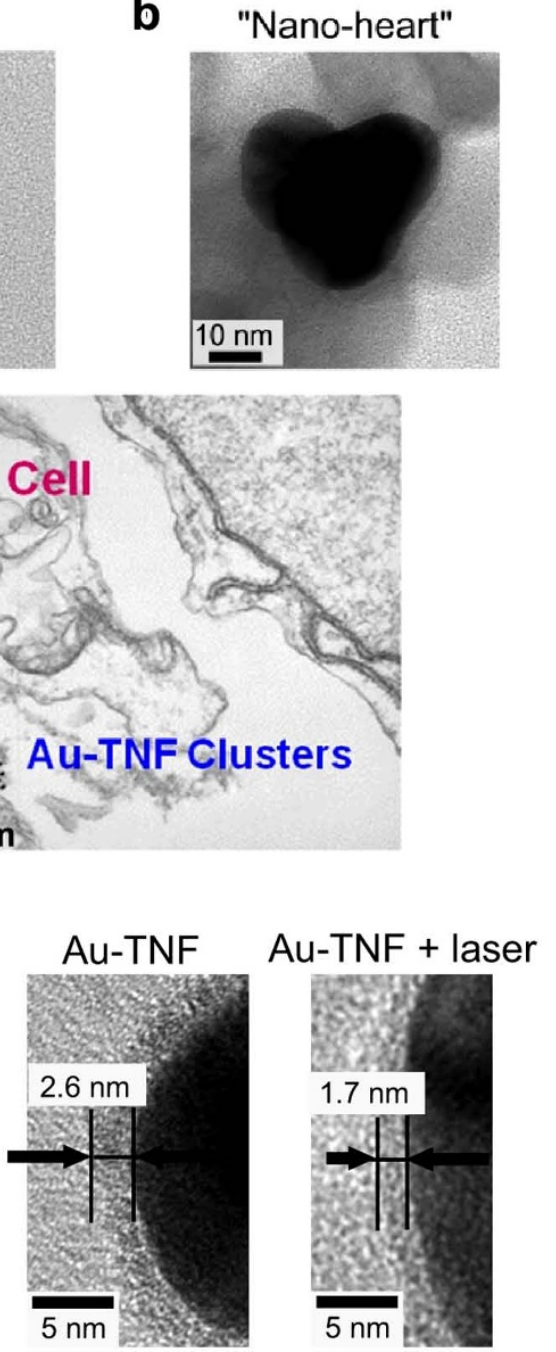

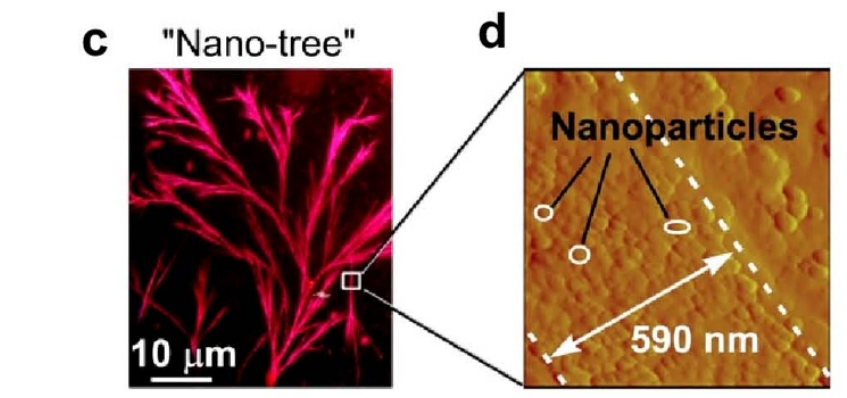

f

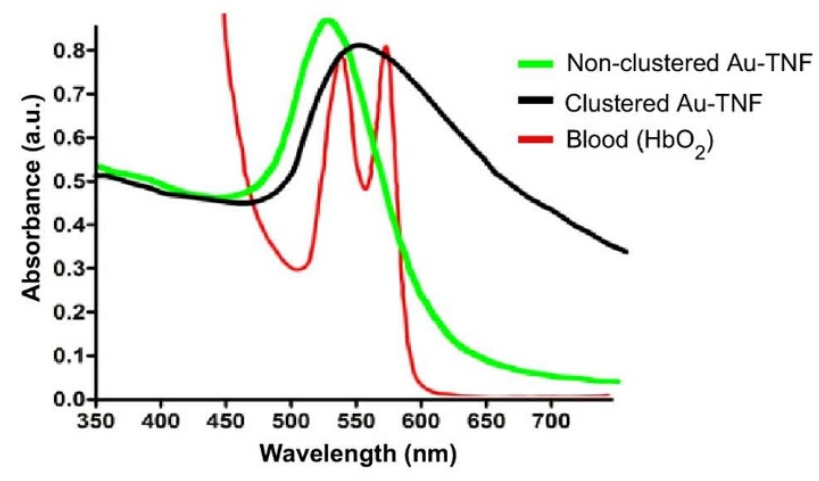

h

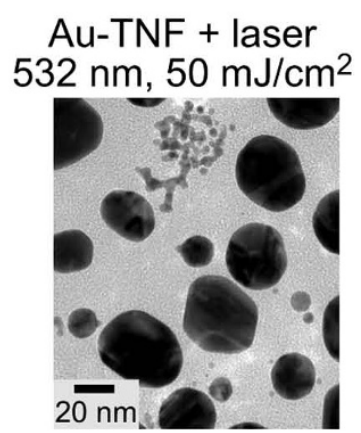

Au-TNF + laser

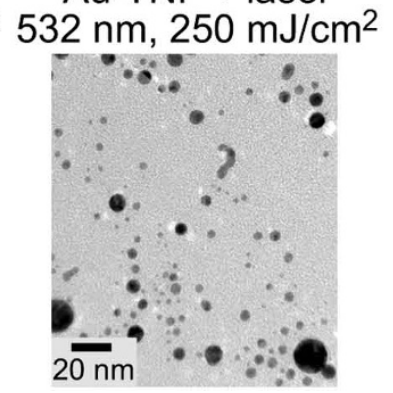

Figure $2 \mid$ Au-TNF nanoparticle characterization. TEM imaging of (a) single nanoparticle and (b) of a small cluster of three nanoparticles ("nanoheart"). (c) Optical image of large nanoparticle cluster ("nano-tree") in dried solution. (d) Atomic force microscopy of nanoparticle cluster fragment corresponding to a white square in (c). (e) Transmission electron microscopy (TEM) image of Au-TNF clusters in $2 \mathrm{H} 11$ cells, $24 \mathrm{~h}$ incubation. (f) Absorption spectra of blood, single, and clustered Au-TNF nanoparticles. (g) TNF release from PEG coating of Au-TNF during laser heating (wavelength, $532 \mathrm{~nm}$; energy fluence, $50 \mathrm{~mJ} / \mathrm{cm}^{2}$ ). (h) Nanoparticle fragmentation by high energy laser pulses.

(in the center and slightly red- and blue-shifted) at relatively low energy (Fig. 3c), while at higher energies PT/PA resonances were more red-shifted (off-center ultrasharp PT/PA resonances).

Thus, clustering of nanoparticles in cells and tissue provides a significant shift of plasmonic resonance to NIR range that may make more effective application of 690-nm therapeutic laser with spherical nanoparticles leading even to nanoparticle fragmentation at moderate laser energy fluence (Fig. 2h). Nonlinear dynamic processes are expected to further enhance efficacy of laser interaction with the core of Au-TNF PT nanodrug.

Cytotoxicity of Au-TNF as a photothermal nanodrug. A cytotoxicity study using 3-(4,5)-dimethylthiahiazo(-z-y1)-3,5-di-phenytetrazoliumromide (MTT) assay revealed the half-maximal (50\%) inhibitory concentration $\left(\mathrm{IC}_{50}\right)$ of Au-TNF for SCK, 4T1-GFP, and $2 \mathrm{H} 11$ cells, at $15 \mu \mathrm{g} / \mathrm{ml}, 10 \mu \mathrm{g} / \mathrm{ml}$ and $10 \mu \mathrm{g} / \mathrm{ml}$, respectively (Supplementary Fig. S5). PT assays based on monitoring the increase in PT signals from cells with nanoparticles (Supplementary Fig. S6, S7) ${ }^{18}$ and TEM (Fig. 2e) both revealed that maximal Au-TNF uptake was in cancer cells (e.g., 40-60\% for SCK) compared to no or low (15-35\%) uptake by mouse red blood cells (RBCs) and white blood cells (WBCs) (Fig. 4a). To explore PT nanodrug properties we compared cancer cell viability in vitro after exposure to Au-TNF alone or combined with laser (Supplementary Fig. S8) using the MTT assay, while varying the incubation time $(0-24 \mathrm{hrs})$ (Fig. $4 \mathrm{~b})$, nanodrug dose $(0-5 \mu \mathrm{g} / \mathrm{mL})$ (Fig. 4c) and laser energy fluence $\left(0-3 \mathrm{~J} / \mathrm{cm}^{2}\right)$ (Fig. $4 \mathrm{~d}$ ). We revealed little to no cytotoxic effects with laser or Au-PEG alone or Au-TNF alone (Figs. 4b, 4c, 4d). However, a laser-induced therapeutic effect with Au-TNF was more significant compared to Au-PEG with laser irradiation (Figs. 4b, 4c, 4d). A maximal difference in therapeutic efficiency was observed after $4 \mathrm{~h}$ incubation with Au-TNF at a concentration of $5 \mu \mathrm{g} / \mathrm{mL}$ and laser energy fluence of $0.5 \mathrm{~J} / \mathrm{cm}^{2}$ (Figs. 4b, 4c). This difference was lower at a longer incubation time (24 h) (Fig. 4b). To exclude the influence of the laser on cell viability, the assay was repeated with Au-TNF or Au-PEG first pre-treated with laser and then added to cells (Supplementary Fig. S9). In this case laser pre-treated Au-TNF at $532 \mathrm{~nm}$ or $690 \mathrm{~nm}$ with energy fluence of $0.5 \mathrm{~J} /$ $\mathrm{cm}^{2}$ also had higher therapeutic effect compared to Au-PEG or AuTNF without laser (Supplementary Fig. S9). Calculations of nanoparticle core temperature and TEM analysis of the polymer layer around it (Supplementary Documents 1, 2 and Figs. S10-S14) suggested that the laser exposure caused release of TNF from the PEG layer at moderate laser fluence, while the core mostly remained intact (Fig. 2g, Supplementary Figs. S13, S14). At high laser pulse 
a

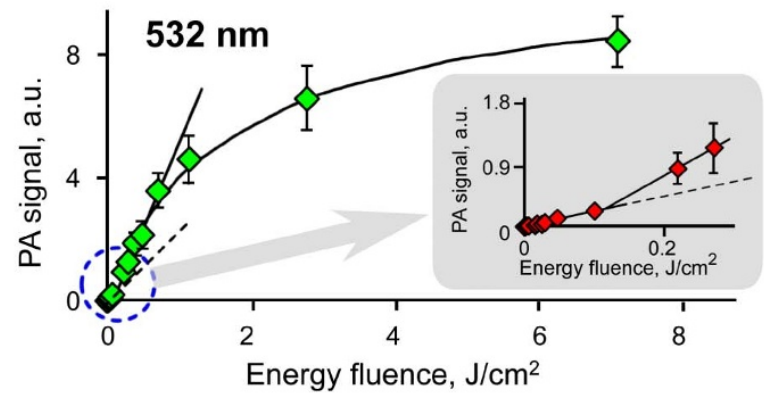

b

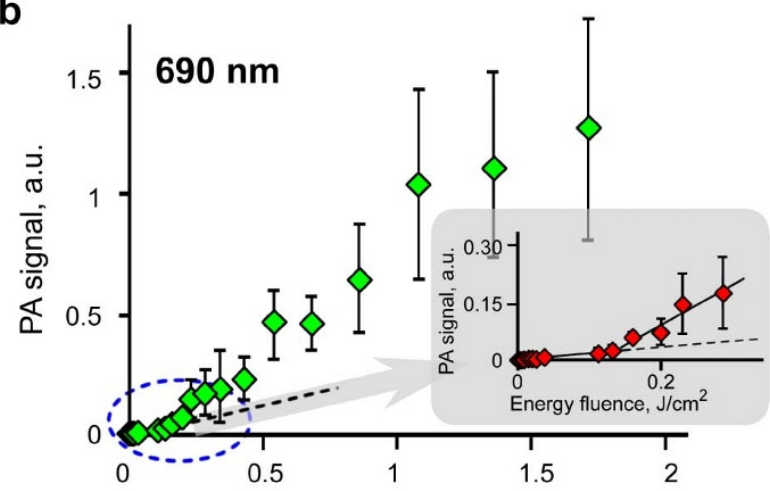

C

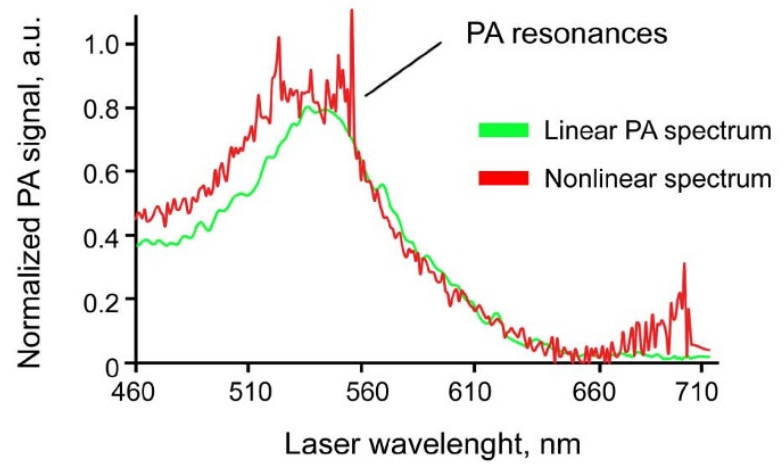

Figure 3 Nonlinear PA dynamics in Au-TNF conjugates. (a), (b) PA signal amplitudes as a function of laser energy fluence at laser wavelengths of $532 \mathrm{~nm}$ (a) and $690 \mathrm{~nm}$ (b). (c) Linear and nonlinear normalized PA spectra of Au-TNF suspension at laser energy fluence of $50 \mathrm{~mJ} / \mathrm{cm}^{2}$ and $500 \mathrm{~mJ} / \mathrm{cm}^{2}$. All PA experiments were performed in $10^{11}$ particles $/ \mathrm{mL} \mathrm{Au}$ TNF solution (120 $\mu \mathrm{m}$ light path), laser beam diameter $50 \mu \mathrm{m}$.

energy we observed melting and fragmentation of the nanoparticles (Fig. 2h, Supplementary Fig. S13). For both moderate and high laser energy levels fragmentation results in formation of spherical small particles with dominant size of size of 3-5 nm at $532 \mathrm{~nm}$ and of 5$10 \mathrm{~nm}$ at $690 \mathrm{~nm}$ (Supplementary Fig. S14).

Pharmacokinetics of Au-TNF. The in vivo experiments were performed using an implanted tumor-window chamber model (Fig. 5a) and imaging of blood vasculature with optical (Figs. 5b, Supplementary Fig. S15, S16) and PA microscopy ${ }^{26-29}$ before and after intravenous (i.v.) injection of $250 \mu \mathrm{g} / \mathrm{kg}$ of Au-TNF. Control and tumor-bearing mice displayed gradual increases in PA image contrast (Figs. 5c, 5d, Supplementary Fig. S15) associated with either increase of nanoparticle concentration in blood flow, or with their immobilization on the vessel walls. High speed PA flow cytometry $(\mathrm{PAFC})^{28,29}$ was applied for real-time monitoring of the nanoparticle depletion kinetics in bloodstream either through window chamber or directly in arterioles of the ear. PAFC revealed immediate appearance of nanoparticles in blood flow followed by a PA signal decrease during 20-40 minutes depending on vessel type and size (Fig. 5e). PA microscopy data correlated well with the data on $\mathrm{Au}-$ TNF concentration in blood and revealed a difference in Au-TNF accumulation kinetics for healthy and tumor vasculature. For control mice the maximum PA signal was observed at 20 min after injection followed by a gradual decrease (Fig. 5f) suggesting temporal immobilization of nanoparticles on the vessels walls and their subsequent release back into the bloodstream. On the contrary, tumor vasculature displayed almost linear growth of signal contrast over several hours post injection (Fig. 5g) indicating nanoparticle accumulation at the tumor site. PA imaging also provided a direct confirmation of nanoparticle clustering (Figs. $5 c$, $5 \mathrm{~d}$, right). Specifically, PA microscopy revealed a homogeneous increase in PA contrast in the vasculature with some randomly distributed PA signals with ultra-high amplitudes appearing $\sim 60-$ $80 \mathrm{~min}$ after the injection. These local high amplitude signals (at least 6-10-fold above blood background) are caused by nonlinear nanobubble related PA signal amplification from strongly absorbing nanoparticle clusters $\mathrm{s}^{23-24}$ and were observed in large numbers in tumor related tissues and rarely in normal vasculature (Supplementary Fig. S15). Presence of Au-TNF clusters in tumor tissues has been confirmed in our work and other studies using TEM and with silver enhancement of gold nanoparticle contrast in histological tumor samples (Fig. 2e, Supplementary Figs. S3, S4). We additionally confirmed the presence of Au-TNF in tumor tissues using X-ray local spectral microanalysis (Supplementary Fig. S4).

Comparison of the data obtained by PA microscopy in static and PA flow cytometry in dynamic conditions revealed that accumulation of nanoparticles in the tumor region could not be directly associated with the fast deposition of nanoparticles immediately after injection when their concentration in blood is maximal. Our data rather indicate that this accumulation takes a relatively long time and is likely related with slower interactions between a low concentration of nanoparticles remaining in the blood flow and tumor vasculature. This was in part confirmed by the observed gradual decrease of PA signals from vessels of healthy tissue in control mice due to release of nanoparticles back into the blood flow (Fig. 5f), while in tumor we observed the steady increase of PA signal from vessels (Fig. 5g). It is not clear if local clustering observed with PA microscopy (Supplementary Fig. S15) was related to pre-existing local vessel damage which increased Au-TNF permeability, or to tumor heterogeneity. We believe that differences in accumulation may be related to the known heterogeneity of tumor response to TNF as revealed by previous work that observed $\mathrm{Au}$-TNF disrupted the tumor vasculature to a different extent in various regions of the same tumor ${ }^{30}$.

Therapeutic application of nanoparticles and laser. Our in vitro therapeutic-related results prompted further study of the PT nanotherapeutic effects on solid tumors using nanosecond clinicallyapproved lasers at wavelengths of $532 \mathrm{~nm}$ and $690 \mathrm{~nm}$. We used similar levels of laser energy fluence in the range of $0.1-1 \mathrm{~J} / \mathrm{cm}^{2}$ at both wavelengths (i.e., 532 and $690 \mathrm{~nm}$ ). As shown in Figure 6a, 6b, treatment with Au-TNF alone showed no obvious difference in tumor regression compared to that of the saline-treated group, whereas the laser irradiation of tumor at $690 \mathrm{~nm}$ after injection of $\mathrm{Au}$-TNF nanoparticles resulted in a significant suppression of tumor growth. The tumor growth delay study illustrated that the control groups (no laser and no drug) and Au-TNF alone group all grew to about $6-8$-fold of the starting volume in 7 days. Tumors treated with $532 \mathrm{~nm}$ laser alone, $690 \mathrm{~nm}$ laser alone, or Au-PEG with $690 \mathrm{~nm}$ laser grew to approximately 3 and 5 -fold of the starting volume by 7 days, respectively. However, in stark contrast, tumor-bearing mice treated with laser at $532 \mathrm{~nm}$ combined with Au-TNF mostly had to be euthanized due to a large amount of damage to the tumor and 


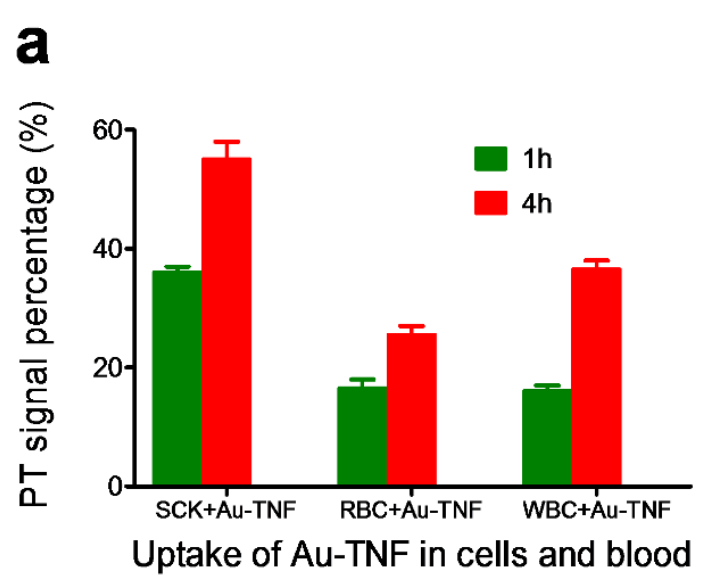

C

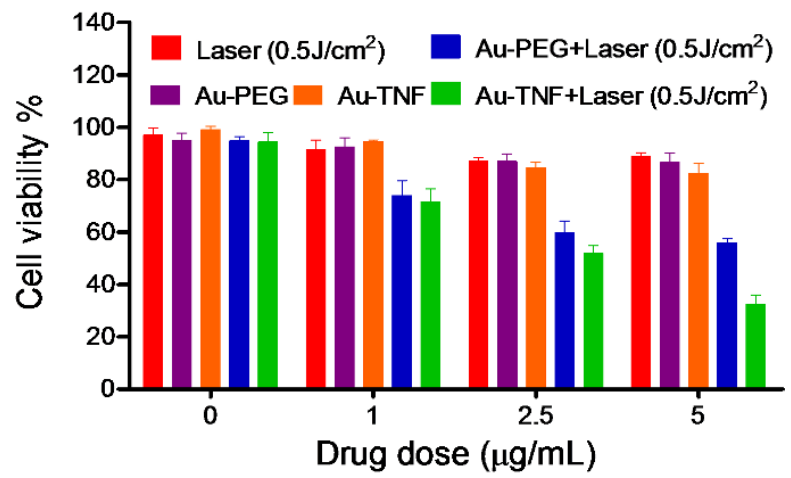

b

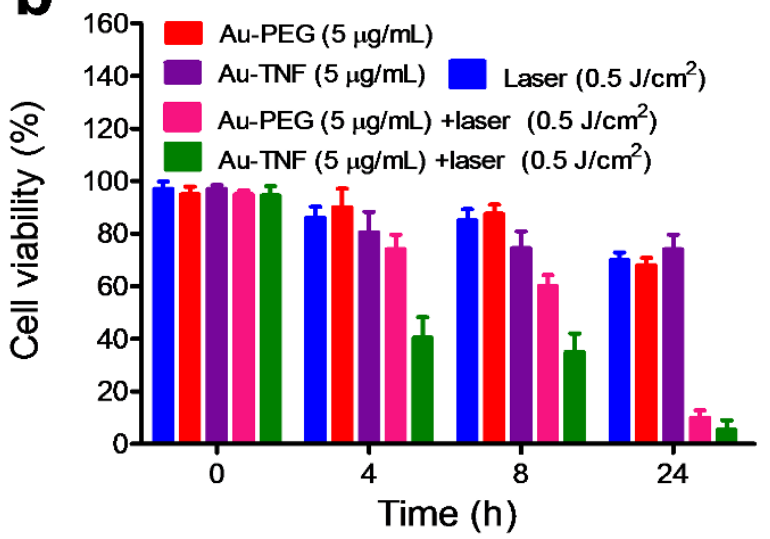

d

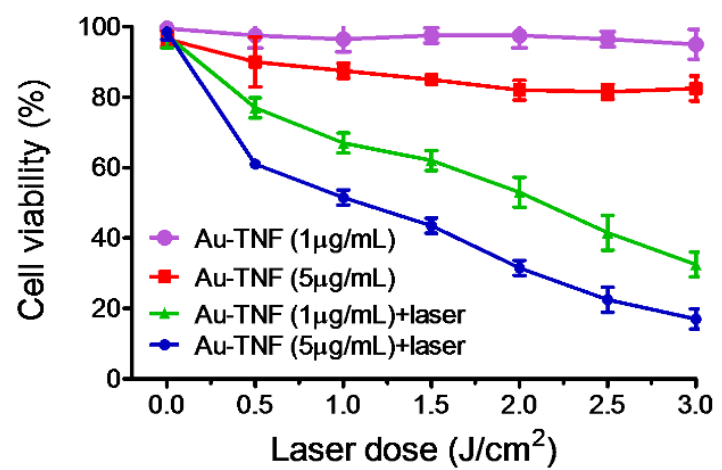

Figure $4 \mid$ In vitro cell-nanoparticle interaction and cytotoxicity of Au-TNF with pulsed laser. (a) Cellular uptake of Au-TNF in SCK cancer cells and murine blood. (b) Cell viability after different therapeutic regimens. (c) Cell viability after photothermal therapy and graded drug concentration. (d) Cell viability after different laser energies for selected Au-TNF concentration. Results are expressed as the mean \pm SD of three replicates of each treatment.

surrounding inflammation of normal tissue in these mice within the first 7 days after treatment (Fig. 6c). While this was an indication of the synergistic effects of laser and Au-TNF treatment, it also made clear that the $532 \mathrm{~nm}$ laser is not a viable option for PT therapy. Therefore, we did an additional experiment to reveal the $532 \mathrm{~nm}$ laser damage of vasculature in a control mouse with no nanoparticles. The laser alone did cause some damage, yet not nearly to the degree that combined Au-TNF and $532 \mathrm{~nm}$ caused (Supplementary Fig. S16). In the case of Au-TNF combined with laser at $690 \mathrm{~nm}$, tumor volume reached no more than 5 fold in the first week after treatment and there was no collateral normal tissue damage. More interestingly, an apparently complete resorption of tumors was observed within 9 days after PT therapy, and no growth in tumor volume was observed for at least 2 weeks (Fig. 6c).

\section{Discussion}

In the present work, we have introduced a novel concept of nanodrug based on synergy of biochemical and physical mechanism actions and verified this concept using Au-TNF conjugates activated by laser-induced PT effect. Taking into account the obtained data and previous findings ${ }^{9,10,17,18,31,32}$ we can suggest that PT effects might lead to 1 ) temperature dependent drug action amplification; 2) ultrafast (picosecond scale) thermal expansion of nanoparticles ${ }^{17}$ accompanied by drug penetrating deeper into tissue; 3 ) generation of PA waves providing possible acoustic-sensitive drug activation; 4) nanobubbles responsible for physical cell damage; 5) nanoparticle explosion ${ }^{17}$ creating small nanoparticle and drug fragments. We cannot exclude that explosive PT phenomena may lead to ultra local formation of highly reactive radicals (e.g., TNF molecule fragments) potentially having higher toxicity (compared to intact TNF and nanoparticles); however this issue requires additional studies.

We found that gold nanosphere conjugates are almost ideal for development as a PT nanodrug using these mechanisms because of mentioned simple fast conjugation procedures, strong controllable PT effects, low cost and low toxicity ${ }^{17-21}$. It is also important that AuTNF conjugates that we used are currently in a phase II trials on humans (as a single therapy with no laser) ${ }^{21}$ emphasizing the clinical relevance of PT nanodrug.

Indeed, the obtained data clearly showed that the combination Au-TNF with laser at $690 \mathrm{~nm}$ in solid tumors for PT therapy has immense potential therapeutic value. This observation further supports our concept of PT nanodrug when targeted with a pulsed laser at a suitable wavelength can provide PT activation of the TNF-associated therapeutic effect and potentially promote the TNF release into the interstitial space and/or cause thermal sensitization of the tumor. This finding is also supported by our data illustrating a lower therapeutic efficiency of PEGylated gold nanospheres of identical size but without TNF even in combination with laser therapy. At the same time, pre-treating Au-TNF solution with laser in the absence of cells appears to liberate TNF and/or create much smaller particles with TNF still attached (Supplementary Fig. S13, S14). This led to a decrease in viability when the irradiated solutions were subsequently incubated with cells (Supplementary Fig. S9). We surmise that this result was associated with laser-released $\mathrm{Au}$-TNF products in solution and was found to be laser energy dependent. Thus, conventional mechanism of pulsed PT therapy associated with laser-induced nanobubble formation ${ }^{9,10}$ for physical tumor cell damage was significantly enhanced here by simultaneous activation of the biochemical TNF action in the tumor. Indeed, our study both in vitro and in vivo clearly 
a

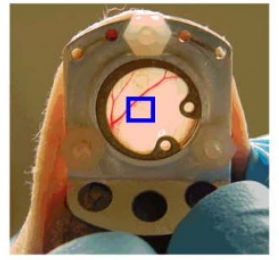

C

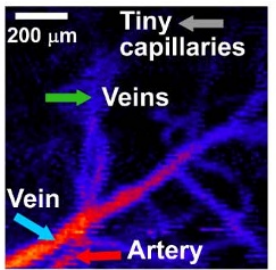

Before

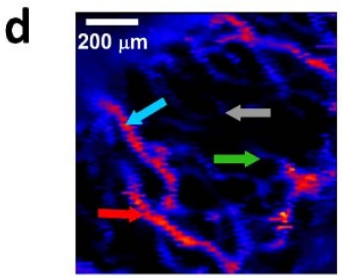

Before

e

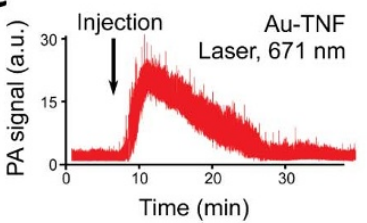

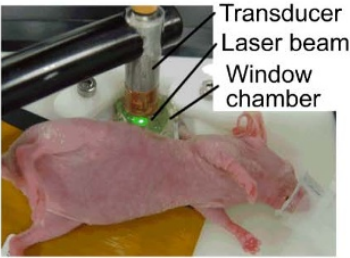
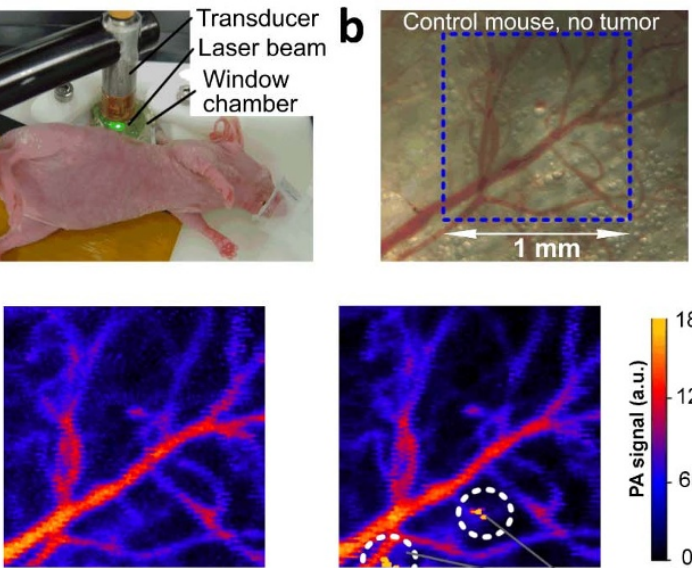

10 min after

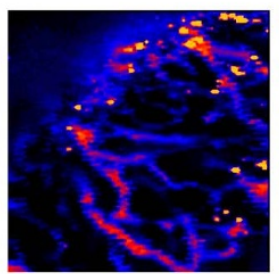

50 min after

f

Au-TNF uptake control mouse

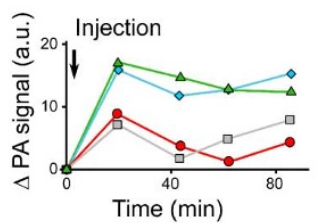

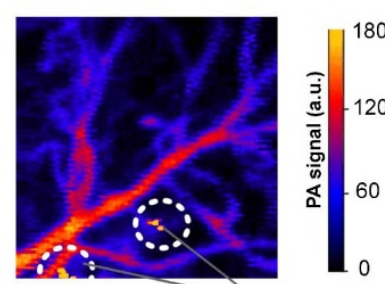

70 min after Aggregates of Au-TNF

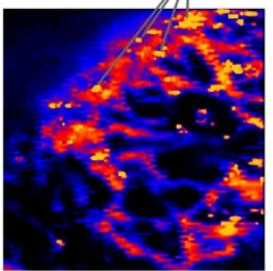

80 min after

g

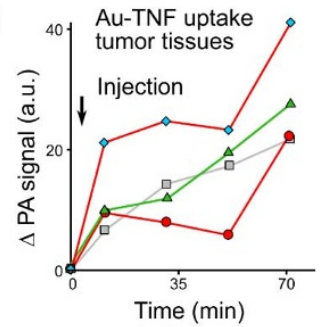

Figure 5 | Au-TNF pharmacokinetics in murine model. (a) Window chamber (left) and PA probe with ultrasound transducer (right). (b) Healthy mouse vasculature in window chamber. (c) PA imaging of healthy tissues before and after i.v. Au-TNF injection. (d) SCK tumor vasculature after i.v. AuTNF injection. Local intensive PA signals correspond to nanoparticle clusters. (e) In vivo PA flow cytometry of Au-TNF nanoparticle kinetics in mouse ear microvessels. (f, g) Increase in PA signal after Au-TNF injection (data from panels $\mathrm{c}$ and $\mathrm{d}$ ) for healthy (f) and tumor bearing ( $\mathrm{g}$ ) mice. Laser parameters for $\mathrm{c}$ and d: wavelength, $532 \mathrm{~nm}$; energy fluence, $50 \mathrm{~mJ} / \mathrm{cm}^{2}$; scanning step size, $10 \mu \mathrm{m}$. Laser parameters for e: $671 \mathrm{~nm}, 100 \mathrm{~mJ} / \mathrm{cm}^{2}$.

suggests high efficiency of PT therapy with Au-TNF conjugated nanoparticles compared to nanoparticles alone.

The efficient laser heating of PT nanodrug is essential for in vivo therapy and TNF activation. We have discovered that even individual Au-TNF conjugates have significantly higher absorbance (4-6-fold and higher) in NIR region compared to the theoretical level calculated for single 30-nm gold spheres (Supplementary Document S1). We revealed that both increased light absorbance and shift of plasmonic resonance from $520-525 \mathrm{~nm}$ to $535-$ $540 \mathrm{~nm}$ were caused by a slightly non-uniform spherical, elongated shape of the nanoparticles (Fig. 2a). Indeed, TEM images show that the ratio between major and minor diameters of Au-TNF varied from 1.1 to 1.3 and even 2.0 for small nanoparticle percent. After correcting the spectral data for Au-TNF, our theoretical calculations predicted that laser heating of single Au-TNF conjugates at $690 \mathrm{~nm}$ even without clustering may activate the nano-drug action (Supplementary Fig. S10, S11, S14). We also experimentally confirmed the release of TNF from the polymer shield of single nanoparticles (Fig. 2g). However, at $690 \mathrm{~nm}$ these phenomena require relatively high laser fluence of $200 \mathrm{~mJ} / \mathrm{cm}^{2}$ and higher.

We observed also evidence of clustering of Au-TNF in tissues using multimodal techniques such as TEM (Supplementary Fig. S4) and real-time PA microscopy (Supplementary Fig. S15). The clustering and associated red spectral shift dramatically increases absorbance contrast of Au-TNF at $690 \mathrm{~nm}$ compared to $532 \mathrm{~nm}$, thus improving PT therapy efficiency and safety due to a reduced (20-fold) blood absorption background at $690 \mathrm{~nm}$ compared to $532 \mathrm{~nm}$. In addition we revealed that for optimized laser parameters, nonlinear dynamic PT phenomena occur. These lead to spectral sharpening and the amplification of PT effects due to nanobubble formation and nanoparticle size and shape modification (e.g., due to its melting) during laser pulse and may play an important role in the synergistic enhancement of PT therapy even for small nanoclusters if not for individual nanoparticles (Fig. 3a-c). According to the obtained data the amplitude of ultrasharp PT resonances at $690 \mathrm{~nm}$ was comparable to that at $532 \mathrm{~nm}$ suggesting that approximately 60-fold and 15-fold amplification of PT effects for spherical and ellipsoidal nanoparticles would occur, respectively. We cannot exclude that these universal nonlinear PT phenomena which we have previously observed in various gold nanoparticles (e.g., nanospheres, rod and shells), chromophores and dyes ${ }^{25}$ may be further enhanced using multiphoton absorption and multi (e.g., second and third) harmonic generation ${ }^{3-37}$. We believe that a combination of all these nonlinear phenomena with the use of slightly ellipsoid nanoparticles and their clustering in cells and tissues could provide a very effective cancer nanotherapy. The presented clinically relevant approach of drug activation through PT heating of a nanoparticle that is progressing through human clinical trials at present can open new options 

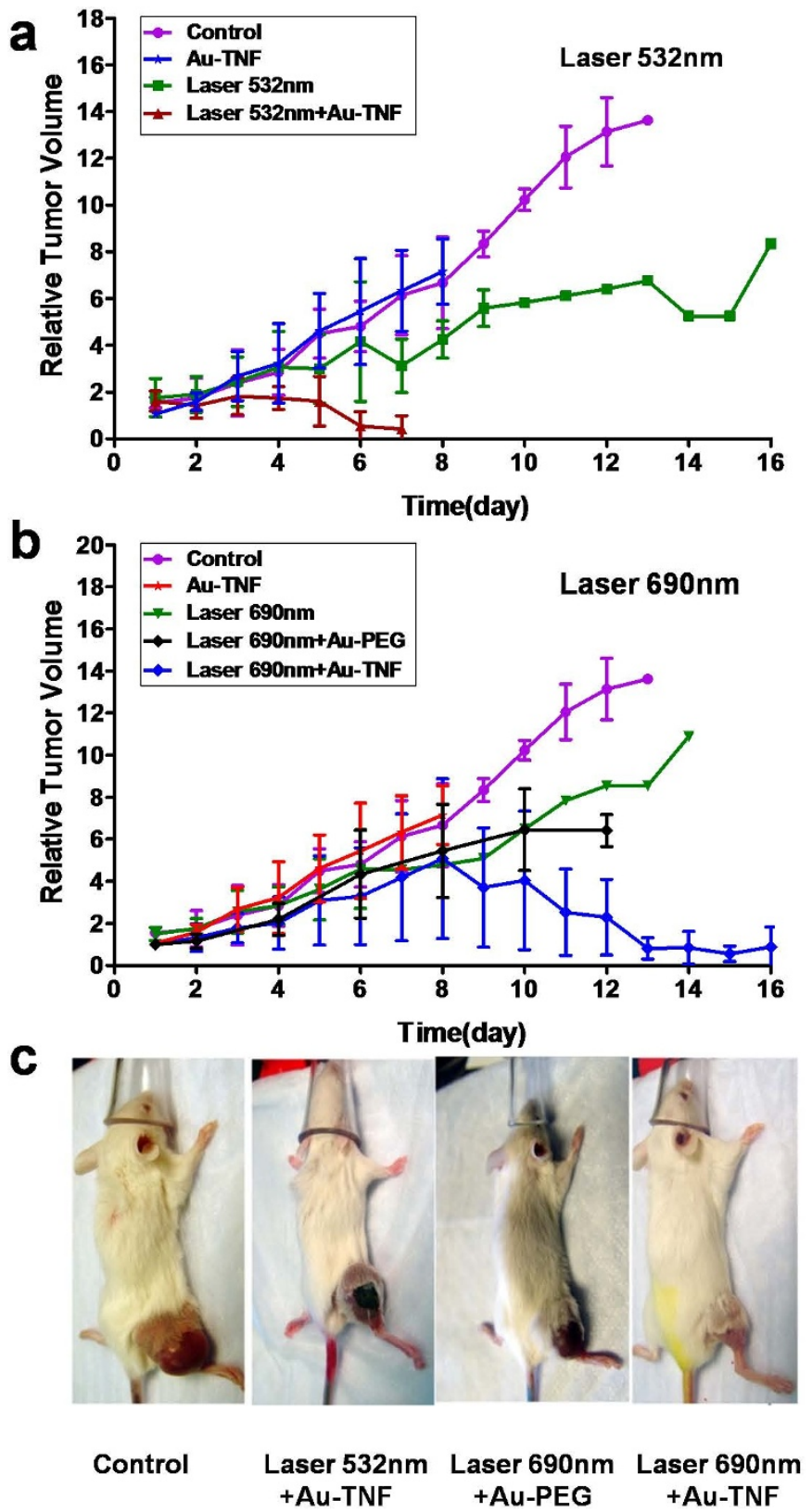

Figure 6 | In vivo anti-tumor effect of Au-TNF nanoparticles activated by pulsed laser at wavelength of $532 \mathrm{~nm}$ and $690 \mathrm{~nm}$. Relative tumor volume increase after treatment with laser alone, and laser with Au-TNF or Au-PEG at laser wavelength of $532 \mathrm{~nm}$ (a) or $690 \mathrm{~nm}$ (b) at $8 \mathrm{~h}$ after an i.v. injection of nanoparticles. (c) Tumor images of different mouse groups. Each group contained 5 mice.

for synergistic cancer treatment using multiple modalities. Moreover, we have demonstrated here that integration of noninvasive PA detection of nanoparticle accumulation in tumor and surrounding tissue followed by $\mathrm{PT}$ therapy using the same laser at different energies is a promising PA-PT nano-theranostic approach.

\section{Methods}

Pegylated Au-TNF nanoparticles. Pegylated Au-TNF of 33-nm were supplied by Cytimmune, Inc and synthesized as previously described ${ }^{32}$. Briefly, $\mathrm{pH}$ adjusted colloidal gold sol (pegylated Au NPs) and TNF at $1 \mu \mathrm{g} / \mathrm{ml}$ were combined and vigorously mixed in a flow through module. The resultant mixture was collected and allowed to stir for $15 \mathrm{~min}$ followed by the addition of the PEGTHIOL $(15 \mu \mathrm{g} / \mathrm{ml}$; Sun BIO Corp, Walnut Creek, CA, USA), and after 15 min of bovine serum albumin (5\% $\mathrm{v} / \mathrm{v}$ at $200 \mu \mathrm{g} / \mathrm{ml}$ in $\mathrm{DIH}_{2} \mathrm{O}$ ). Colloidal-gold-bound TNF in each preparation was separated from free TNF by diafiltration through a 50,000 MWCO BIOMAX diafiltration cartridge (Millipore Corporation, Chicago, IL, USA). TNF content was analyzed in both the permeate (i.e., free TNF) and in the retentate (TNF-bound colloidal gold). The colloidal gold was sterile filtered through a $0.22 \mu \mathrm{m}$ filter and frozen at $-80^{\circ} \mathrm{C}$ for storage or lyophilization. Shah et $\mathrm{al}^{30}$ performed a detailed study on the effects of pegylation and TNF coating on blood and tissue interactions of the particle after injection. In addition, this study describes in detail the basic approach that the parent company has taken in synthesizing the gold-TNF agent.

Cell lines and culture. Murine SCK and 4T1-GFP breast carcinomas and 2H11 lymphatic endothelial cells were obtained from the department of Radiation Oncology, Dr. Alexander Asea and ATCC, respectively. The cell lines were maintained in RPMI 1640 (Gibco) or DMEM (Gibco) medium, supplemented with $10 \%$ bovine calf serum (Hyclone) or 10\% Fetal Bovine Serum (Hyclone), $2 \mathrm{mM} \mathrm{L-}$ glutamine, 100 units $/ \mathrm{ml}$ penicillin and $100 \mathrm{mg} / \mathrm{ml}$ streptomycin (Cellgro) in a humidified atmosphere of $5.0 \% \mathrm{CO}_{2}$ at $37^{\circ} \mathrm{C}$. The medium was replaced with fresh medium three times per week until the cells grew $80-90 \%$ confluence. Cell lines at final density of $1 \times 10^{5}$ cells $/ \mathrm{mL}$ were cultured in 96 -well plates $(100 \mu \mathrm{L}$ per cell) for the MTT assay, or in 6-well plates ( $2 \mathrm{~mL}$ per well) for studies of the trypan blue assay.

MTT assay for cell viability. Cell viability was assessed by the MTT assay based on the reduction of MTT by mitochondrial dehydrogenases of viable cells to a purple formazon product. Briefly, the above mentioned cell lines $\left(1 \times 10^{4} /\right.$ well $)$ were plated in $100 \mu \mathrm{L}$ of medium/well in 96-well plates. After overnight incubation in the culture medium for $70-80 \%$ confluence, the cells were treated with different concentrations $(1-25 \mu \mathrm{g} / \mathrm{mL})$ of Au-TNF (Cytimmune sciences, Inc., Rockville, MD) in fresh medium for $24 \mathrm{~h}$. Subsequently, the cells were washed with $200 \mu \mathrm{L}$ PBS, and incubated with $50 \mu \mathrm{g}$ MTT (sigma) per well for another $4 \mathrm{~h}$ in the medium without phenol red and serum. The MTT-formazan formed by metabolically viable cells was dissolved in $100 \mu \mathrm{L}$ of DMSO and shaken for $10 \mathrm{~min}$. The absorbance was then measured on an ELISA reader at a test wavelength of $570 \mathrm{~nm}$. Each test was repeated at least three times. The concentration of the nanodrug which gives the $50 \%$ growth inhibition value corresponds to $\mathrm{IC}_{50}$.

Trypan blue assay for cell viability. The above-mentioned cell lines after incubation at $37^{\circ} \mathrm{C}$ until reaching $70-80 \%$ confluence in 6-well plates were treated with fresh serum-free medium $(100 \mu \mathrm{L})$ containing the Au-PEG and Au-TNF nanodrugs at various concentrations $(1-5 \mu \mathrm{g} / \mathrm{mL})$ for 4 hours. The cells were trypsinized from the 6-well plate, counted, and then diluted in PBS until a $1 \mathrm{~mL}$ sample contained $\sim 1$ million cells. The samples were then irradiated for durations (60 s) with a laser (532 nm, Nd:YAG) at $10 \mathrm{~Hz}$ corresponding to varying energy densities of $0.5-3.0 \mathrm{~J} /$ $\mathrm{cm}^{2}$. After the irradiation, the cell lines were returned to the incubator for 4-24 hours. Viability was assayed before and after treatment using a $1: 1$ solution of trypan blue dye (sigma) and counting cells taking up the dye as membrane compromised under the microscope.

Animal and experimental design in vivo. A/I mice (18-22 g body weight, 6-8 weeks old) were maintained under consistent temperature, humidity and light/dark cycle conditions and fed with standard laboratory food and water ad libitum. Murine mammary cancer cells (SCK) were trypsinized and resuspended in PBS. A total of $3 \times$ $10^{5}$ cells were subcutaneously injected in the hindlimb of nu/nu AJ mice weighing $20 \mathrm{~g}$. Experiments were performed after 5-7 days when a tumor diameter of 7-8 mm was obtained. Tumor-bearing mice were randomized into seven experimental groups, each with 5 mice. Group I was the normal saline control, group II and III was Laser $532 \mathrm{~nm}\left(1 \mathrm{~J} / \mathrm{cm}^{2}\right)$ and Laser $690 \mathrm{~nm}$ alone $\left(1 \mathrm{~J} / \mathrm{cm}^{2}\right)$ controls, group IV-VIII was treated with Au-TNF alone $(250 \mu \mathrm{g} / \mathrm{kg})$, Au-TNF $(250 \mu \mathrm{g} / \mathrm{kg})+$ Laser $532 \mathrm{~nm}$ or $690 \mathrm{~nm}\left(1 \mathrm{~J} / \mathrm{cm}^{2}\right)$ and Au-PEG $(250 \mu \mathrm{g} / \mathrm{kg})+$ Laser $690 \mathrm{~nm}\left(1 \mathrm{~J} / \mathrm{cm}^{2}\right)$, respectively. The group IV-VIII tumor-bearing mice were injected with Au-TNF and Au-PEG via tail vein at a dose of $0.1 \mathrm{~mL} / 20 \mathrm{~g}$ bodyweight for 8 hours before laser $532 \mathrm{~nm}$ or $690 \mathrm{~nm}\left(1 \mathrm{~J} / \mathrm{cm}^{2}\right)$ irradiation for 60 seconds.

Animal survival and tumor measurement. Tumor-bearing mice after $\mathrm{Au}-\mathrm{TNF}, \mathrm{Au}-$ PEG and laser treatment groups were closely observed for side effects or evidence of toxicity. Tumor size was measured once every 2 days in two perpendiculars and tumor volume was estimated by measuring the outside diameter of the tumor with a calipers and using the formula $\mathrm{a}^{2} \mathrm{~b} / 2$ where $a$ and $b$ are the shorter and longer diameters, respectively. The body weight of the animals was measured three times a week at the same time as the tumor dimension measurement and the mortality. At the end of the experiment, the animals were sacrificed and the tumors were dissected and weighed. The tumor growth inhibition rates were then determined. All studies were approved by the University of Arkansas for Medical Sciences Institutional Animal Care and Use Committee.

Au-TNF uptake by cells TEM analysis. TEM was used to verify the uptake of gold nanoparticles by the cancer and endothelial cells. The 2H11 and 4T1-GFP cells were harvested at 1, 4, 24 hours post Au-TNF treatment. They were postfixed in $2 \%$ osmium tetraoxide in the buffer for $2 \mathrm{~h}$, washed and dehydrated in graded concentrations of ethanol and propylene oxide. Cell samples were prepared and analyzed by TEM (JEM-2100F, JEOL USA, Peabody, Massachusetts, USA) with an accelerating voltage of $80 \mathrm{kV}$.

AuNPs TEM analysis. TEM was used to determine size, shape and polymer coating of the AuNPs-PEG particles. AuNPs-PEG solution was air dried for 15 minutes on 
holey-carbon coated copper grids. In order to discriminate between the Au nanocore and the surrounding shell, the grid was positively stained by $2 \%$ of uranyl acetate dissolved in $70 \%$ ethanol for $30 \mathrm{~s}$ followed by triple washing with distilled water for 10 seconds each. The average particle size and particle size distribution of each sample were determined by EMAN1 and Image J software public domain software from NIH (rsb.info.nih.gov/ij/). The equivalent circular diameter of each particle was

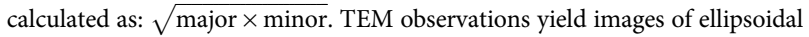
particles as ellipses. The "major" is the longest diameter of an ellipse, while "minor" is the shortest diameter of an ellipse. The mean and standard deviation of the particles" diameters were obtained after measuring over 100 particles in random fields of view for each set of samples.

Photoacoustic (PA) instrument. The PA scanning microscope was built on the technical platform of an invert microscope (model IX81, Olympus America, Center Valley, PA), with incorporated conventional fluorescent, and transmission digital microscope (TDM) modules, and a tunable laser-based optical parametric oscillator (OPO, Opolette HR 355 LD, OPOTEK, Carlsbad, CA) with the following parameters: spectral range of 410-2,200 nm; pulse width, $5 \mathrm{~ns}$; pulse repetition rate, $100 \mathrm{~Hz}$; line width, $\sim 0.5 \mathrm{~nm}$; pulse energy up to $2 \mathrm{~mJ}$; a fluence range, $1-10^{4} \mathrm{~mJ} / \mathrm{cm} 2$; and pulse energy stability, 3-5\%. Energy of each OPO pulse was controlled by energy meter (PE10-SH, OPHIR, Logan, UT). 2.25 MHz ultrasound transducer (V323-SU, Panametrics NDT Inc.) was used to acquire PA signals from the sample in transmission mode (on top of the skin above vessels). The amplified ( $50 \mathrm{kHz}-5 \mathrm{MHz}$ bandwidth amplification, $54 \mathrm{~dB}$ gain, model 5662, Panametrics NDT Inc.) signal was acquired by a digitizer (PCI-5124, National Instruments, Austin, TX), which also controlled sample scanning by a two-dimensional (2 D; X-Y) translation stage (H117 ProScan II, Prior Scientific, Rockland, MA). The intensity of each pixel of PA image represents the amplitude of the PA signal from this location. The ultimate lateral resolution of PAM was determined by the focal spot size of the pump laser beam, and for $10 \times$ objective the diffraction limited resolution of $1.1 \mu \mathrm{m}$ was achieved for $532 \mathrm{~nm}$ excitation.

To verify incubation of cells with Au-TNF and PA data, the PA setup was integrated with PT microscope module ${ }^{38}$. In PT thermal-lens mode, a pump (OPO) laserinduced temperature-dependent variations of the refractive index caused an unfocused collinear He-Ne laser probe beam (633 nm, $1.4 \mathrm{~mW}$ ), and, hence, a reduction in the beam's intensity at its center. This was detected by a photodiode with a pinhole (referred to as PT signals). PT signals demonstrate an initial peak associated with rapid, picosecond-scale heating of nanoparticles and with a slower, nano- to microsecond-scale exponential tail corresponding to sample cooling. In nonlinear mode, laser-induced nanobubbles around overheating nanoparticles lead to the appearance of sharp negative peaks associated with refraction and scattering of the probe beam on the nanobubbles.

1. Agarwal, A., Mackey, M. A., El-Sayed, M. A. \& Bellamkonda, R. V. Remote triggered release of doxorubicin in tumors by synergistic application of thermosensitive liposomes and gold nanorods. ACS Nano 5, 4919-4926 (2011).

2. Dreaden, E. C. \& El-Sayed, M. A. Detecting and destroying cancer cells in more than one way with noble metals and different confinement properties on the nanoscale. Chem. Res. 45, 1854-65 (2012).

3. Farokhzad, O. C. \& Langer, R. Impact of nanotechnology on drug delivery. ACS Nano 3, 16-20 (2009).

4. Ferrari, M. Cancer nanotechnology: opportunities and challenges. Nat. Rev. Cancer 5, 161-171 (2005).

5. Koo, O. M., Rubinstein, I. \& Onyuksel, H. Role of nanotechnology in targeted drug delivery and imaging: a concise review. Nanomedicine: Nanotechnol, Biology and Medicine 1, 193-212 (2005).

6. Nishiyama, N. Nanomedicine: nanocarriers shape up for long life. Nat. Nanotechnol. 2, 203-204 (2007).

7. Peer, D., Karp, J. M., Hong, S., Farokhzad, O. C., Margalit, R. \& Langer, R. Nanocarriers as an emerging platform for cancer therapy. Nat. Nanotechnol. 2, 751-760 (2007).

8. Hirsch, L. R., Stafford, R. J., Bankson, J. A., Sershen, S. R., Rivera, B., Price, R. E., Hazle, J. D., Halas, N. J. \& West, J. L. Nanoshell-mediated near-infrared thermal therapy of tumors under magnetic resonance guidance. P. Natl. Acad. Sci. 100, 13549-13554 (2003).

9. Zharov, V. P., Galitovsky, V. \& Viegas, M. Photothermal detection of local thermal effects during selective nanophotothermolysis. Appl. Phys. Lett. 83, 4897-4899 (2003)

10. Zharov, V. P., Kim, J. W., Curiel, D. T. \& Everts, M. Self-assembling nanoclusters in living systems: application for integrated photothermal nanodiagnostics and nanotherapy. Nanomedicine: Nanotechnol, Biology and Medicine 1, 326-345 (2005).

11. Chakravarty, P., Qian, W., El-Sayed, M. A. \& Prausnitz, M. R. Delivery of molecules into cells using carbon nanoparticles activated by femtosecond laser pulses. Nat. Nanotechnol. 5, 607-611 (2010).

12. Farma, J. M., Puhlmann, M., Soriano, P. A., Cox, D., Paciotti, G. F., Tamarkin, L. \& Alexander, H. R. Direct evidence for rapid and selective induction of tumor neovascular permeability by tumor necrosis factor and a novel derivative, colloidal gold bound tumor necrosis factor. Int. J. Cancer 120, 2474-2480 (2007).
13. Shenoi, M. M., Shah, N. B., Griffin, R. J., Vercellotti, G. M. \& Bischof, J. C. Nanoparticle preconditioning for enhanced thermal therapies in cancer. Nanomedicine 6, 545-563 (2011).

14. Goel, R., Shah, N., Visaria, R., Paciotti, G. \& Bischof, J. Biodistribution of TNFalpha-coated gold nanoparticles in an in vivo model system. Nanomedicine 19, 401-410 (2009)

15. Visaria, R. K., Griffin, R. J., Williams, B. W., Ebbini, E. S., Paciotti, G. F., Song, C. W. \& Bischof, J. C. Enhancement of tumor thermal therapy using gold nanoparticle-assisted tumor necrosis factor- $\alpha$ delivery. Mol. Cancer. Ther. 5, 1014-1020 (2006).

16. Goel, R., Swanlund, D., Coad, J., Paciotti, G. \& Bischof, J. TNF-[alpha] based accentuation of cryoinjury for the treatment of prostate cancer. Cryobiology $\mathbf{5 5}$ 360-360 (2007)

17. Letfullin, R. R., Joenathan, C., George, T. F. \& Zharov, V. P. Laser-induced explosion of gold nanoparticles: potential role for nanophotothermolysis of cancer. Nanomedicine 1, 473-480 (2006).

18. Zharov, V. P., Galitovskaya, E. N., Jonson, C. \& Kelly, T. Synergistic enhancement of selective photothermolysis with gold nanoclusters: potential for cancer therapy. Laser. Surg. Med. 37, 219-226 (2005).

19. Boisselier, E. \& Astruc, D. Gold nanoparticles in nanomedicine: preparations imaging, diagnostics, therapies and toxicity. Chem. Soc. Rev. 38, 1759-1782 (2009).

20. Dykman, L. \& Khlebtsov, N. Gold nanoparticles in biomedical applications: recent advances and perspectives. Chem. Soc. Rev. 41, 2256-2282 (2012).

21. Libutti, S. K., Paciotti, G. F., Byrnes, A. A., Alexander, H. R. Jr., Gannon, W. E., Walker, M., Seidel, G. D., Yuldasheva, N. \& Tamarkin, L. Phase I and pharmacokinetic studies of CYT-6091, a novel PEGylated colloidal gold-rhTNF nanomedicine. Clin. Cancer Res. 16, 6139-6149 (2010).

22. Huang, X., Jain, P. K., El-Sayed, I. H. \& El-Sayed, M. A. Plasmonic photothermal therapy (PPTT) using gold nanoparticles. Laser. Med. Sci. 23, 217-228 (2008).

23. Galanzha, E. I., Shashkov, E. V., Kelly, T., Kim, J. W., Yang, L. \& Zharov, V. P. In vivo magnetic enrichment and multiplex photoacoustic detection of circulating tumour cells. Nat. Nanotechnol. 4, 855-860 (2009).

24. Kim, J. W., Galanzha, E. I., Shashkov, E. V., Moon, H. M. \& Zharov, V. P. Golden carbon nanotubes as multimodal photoacoustic and photothermal high-contrast molecular agents. Nat. Nanotechnol. 4, 688-694 (2009).

25. Zharov, V. P. Ultrasharp nonlinear photothermal and photoacoustic resonances and holes beyond the spectral limit. Nat. Photon. 5, 110-116 (2011).

26. Galanzha, E. I. \& Zharov, V. P. Photoacoustic flow cytometry. Methods 57, 280-296 (2012)

27. Wang, L. V. \& Hu, S. Photoacoustic tomography: in vivo imaging from organelles to organs. Science 335, 1458-1462 (2012).

28. Zharov, V. P., Galanzha, E. I., Shashkov, E. V., Khlebtsov, N. G. \& Tuchin, V. V. In vivo photoacoustic flow cytometry for monitoring of circulating single cancer cells and contrast agents. Ops. Lett. 31, 3623-3625 (2006).

29. Tuchin, V. V., Tárnok, A. \& Zharov, V. P. In vivo flow cytometry: A horizon of opportunities. Cytometry Part A 79, 737-745 (2011).

30. Shah, N. B., Vercellotti, G. M., White, J. G., Fegan, A., Wagner, C. R. \& Bischof, J. C. Blood-nanoparticle interactions and in vivo biodistribution: Impact of surface PEG and ligand properties. Mol. Pharm. 9, 2146-2155 (2012).

31. Pustovalov, V., Smetannikov, A. \& Zharov, V. P. Photothermal and accompanied phenomena of selective nanophotothermolysis with gold nanoparticles and laser pulses. Laser. Phys. Letts. 5, 775-792 (2008).

32. Paciotti, G. F., Myer, L., Weinreich, D., Goia, D., Pavel, N., McLaughlin, R. E. \& Tamarkin, L. Colloidal gold: a novel nanoparticle vector for tumor directed drug delivery. Drug Deliv. 11, 169-183 (2004).

33. Yelin, D., Oron, D., Thiberge, S., Moses, E. \& Silberberg, Y. Multiphoton plasmonresonance microscopy. Opt. Express 11, 1385-91 (2003).

34. Nappa, J., Russier-Antoine, I., Benichou, E. H. \& Brevet, P. F. Second harmonic generation from small gold metallic particles: from the dipolar to the quadrupolar response. J. Chem. Phys. 125, 184712 (2006).

35. Antoine, R., Brevet, P. F., Girault, H. H., Bethellb, D. \& Schiffrinb, D. J. Surface plasmon enhanced non-linear optical response of gold nanoparticles at the air/ toluene interface. Chem. Commun. 19, 1901-1902 (1997).

36. Hari, M., Santhi, A. J., Nithyaja, B., Matthew, S., Kumar, R., Mishra, G., Yadhav, R. R., Radhakrishnan, P. \& Nampoori, V. P. N. Linear and nonlinear optical properties of gold nanoparticles stabilized with polyvinyl alcohol. J. Nonlinear. Optic. Phys \& Mat. 20, 467-475 (2011).

37. Huang, X., Qian, W., El-Sayed, I. H. \& El-Sayed, M. A. The potential use of the enhanced nonlinear properties of gold nanospheres in photothermal cancer therapy. Laser. Surg. Med. 39, 747-753 (2007)

38. Nedosekin, D. A., Galanzha, E. I., Ayyadevara, S., Shmookler, R. R. J. \& Zharov, V. P. Photothermal confocal spectromicroscopy of multiple cellular chromophores and fluorophores. Biophys. J. 102, 672-81 (2012).

\section{Acknowledgements}

This work was performed in the University of Arkansas for Medical Sciences and was supported in part by the National Institute of Health (NIH) grant R01CA44114 (to RJG); R01EB000873, R01CA131164, R01EB009230, R21CA139373, the National Science Foundation (NSF) grant DBI-0852737, and the Department of Defense (DoD) grant: 
W81XWH-11-1-0123 (to VPZ); the NSF grant CMMI-1235100 and Arkansas Biosciences Institute (to J-WK); China Scholarship Council (CSC)Project (No. 2010665505) (to JS). We thank to Cytimmune, Inc for providing Au-TNF nanoparticles, Stephen Foster for help with cell culturing, Evgeny Shashkov for obtaining optical nanoparticle images, Scott Ferguson for assistance with lasers, and Dr. Fumiya Watanabe for the assistance with the electron microscopy studies.

\section{Author contributions}

Conceived and designed the experiments: VPZ, RJG, DAN and JS. Performed the experiments: JS, NK, DAN, VPZ, RJG and TM. Preparation of figures: JS, VPZ, EIG, NK, DAN, RJG and J-WK. Data acquisition: JS, NK, DAN, TM, AB and J-WK. Analyzed the data: JS, NK, DAN, TM, AB and J-WK. Manuscript preparation and writing: JS and VPZ. Manuscript editing: JS, NK, DAN, VPZ, J-WK and RJG. All authors reviewed the manuscript.

\section{Additional information}

Supplementary information accompanies this paper at http://www.nature.com/ scientificreports

Competing financial interests: The authors declare no competing financial interests. License: This work is licensed under a Creative Commons Attribution-NonCommercial-NoDerivs 3.0 Unported License. To view a copy of this license, visit http://creativecommons.org/licenses/by-nc-nd/3.0/

How to cite this article: Shao, J. et al. Photothermal nanodrugs: potential of TNF-gold nanospheres for cancer theranostics. Sci. Rep. 3, 1293; DOI:10.1038/srep01293 (2013). 